\title{
Psychometric Properties of the Spanish Version of the Nonattachment Scale (NAS) and Its Relationship with Mindfulness, Decentering, and Mental Health
}

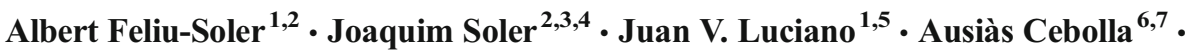 \\ Matilde Elices $^{2,3,8}$ • Marcelo Demarzo ${ }^{9}$. Javier García-Campayo ${ }^{5,10}$
}

(C) Springer Science+Business Media New York 2016

\begin{abstract}
Nonattachment is a Buddhism-rooted construct, which can be defined as the relative absence of fixation on ideas, images, or sensory objects, as well as an absence of internal pressure to get, hold, avoid, or change circumstances or experiences. The present study was aimed at exploring the psychometric properties of the Spanish version of the Nonattachment Scale (NAS) and at delimitating the relationship between the NAS and measures of mindfulness, decentering, and negative emotional symptoms. Pooling the NAS data from Spanish meditators $(n=335)$ and nonmeditators $(n=270)$, we performed an exploratory factor analysis and then estimated the fit of two competing models (one-factor model vs. one-factor model + method effects) via confirmatory factor analysis. Data analyses showed that the Spanish version of the NAS is unifactorial in nature and has excellent internal consistency. As expected, high (positive) significant correlations were found between NAS and mindfulness and decentering measures, as well as high significant
\end{abstract}

Juan V. Luciano

jvluciano@pssjd.org

1 Teaching, Research, and Innovation Unit, Parc Sanitari Sant Joan de Déu, Fundació Sant Joan de Déu, C/Dr. Antoni Pujadas 42, 08830, Sant Boi de Llobregat Barcelona, Spain

2 Centro de Investigación Biomédica en Red de Salud Mental (CIBERSAM), Madrid, Spain

3 Servei de Psiquiatria, Hospital de la Santa Creu i Sant Pau, Institut d'Investigació Biomèdica-Sant Pau (IIB-SANT PAU), Barcelona, Spain

4 Departament de Psicologia Clínica i de la Salut, Universitat Autònoma de Barcelona, Barcelona, Spain (negative) relations between NAS and depression, anxiety, and stress scores. Positive relations were also observed between NAS and variables of meditation practice. Large differences among the meditative, nonmeditative, and clinical groups $(n=39$ patients with borderline personality disorder) were observed regarding NAS scores. Scores on the NAS were significant predictors of negative emotional symptoms (depression, anxiety, and stress) and, especially, resilience. Furthermore, NAS provided a unique contribution in the regression models, going beyond mindfulness facets and decentering. In conclusion, the Spanish version of the NAS is a psychometrically sound instrument with a promising future in the field of mindfulness and meditation research and in clinical settings.

Keywords Nonattachment Scale $\cdot$ Decentering $\cdot$ Mental health $\cdot$ Mindfulness $\cdot$ Resilience

5 Primary Care Prevention and Health Promotion Research Network (RedIAPP), Barcelona, Spain

6 Departament de Personalitat, Avaluació i Tractaments Psicològics, Universitat de València, Valencia, Spain

7 CIBER de Fisiopatología de la obesidad y la nutrición (CIBEROBN), Santiago de Compostela, Spain

8 Programa de Cognición, Facultad de Psicología, Instituto de Fundamentos y Métodos en Psicología, Universidad de la República, Montevideo, Uruguay

9 Mente Aberta - Brazilian Center for Mindfulness and Health Promotion, Department of Preventive Medicine, Federal University of Sao Paulo (UNIFESP), Sao Paulo, Brazil

10 University of Zaragoza and Instituto Aragonés de Ciencias de la Salud, Zaragoza, Spain 


\section{Introduction}

The construct of nonattachment is rooted on Buddhist Psychology and can be defined as the relative absence of fixation on ideas, images, or sensory objects, as well as an absence of internal pressure to get, hold, avoid, or change circumstances or experiences, without defensive detachment or disconnection from one's thoughts and feelings (Sahdra et al. 2010). Recently, Sahdra et al. $(2015$, p. 2) also defined nonattachment in positive terms "as a flexible, balanced way of relating to one's experiences without clinging to or suppressing them."

Nonattachment is thought to encourage psychological flexibility, nonreactivity, objective perception, compassion, and disengaging from difficult emotions and to reduce selfishness (Sahdra et al. 2010). This construct has been inversely related to mental disturbances including dissociation, alexithymia, external locus of control, and avoidance of intimacy in relationships (Sahdra et al. 2010). Conversely, nonattachment has been positively associated with salutary outcomes, such as mindfulness, acceptance, nonreactivity, self-compassion, autonomy, social connectedness, empathy, generosity, wellbeing (Sahdra et al. 2010), and prosocial behavior (Sahdra et al. 2015).

According to one of the most parsimonious definitions of mindfulness as it is conceptualized in Western psychotherapeutic approaches (Bishop et al. 2004), mindfulness may be operationalized as a two-component construct: (1) selfregulation of attention and (2) attitude of openness and acceptance to the present-moment experience. This latter element of mindfulness seems to be implicit in nonattachment, as nonattachment entails an attitude of openness, a letting go of the pressure to get, hold, avoid, or change circumstances. Furthermore, nonattachment seems to be also highly overlapped with the construct of decentering, defined as the ability to observe one's thoughts and feelings in a detached manner, as temporary events in the mind, as neither necessarily true nor reflections of the self (Safran and Segal 1990), and both constructs seem very close to the definition of equanimity ("even-minded mental state or dispositional tendency toward all experiences or objects, regardless of their affective valence (pleasant, unpleasant or neutral) or source"; Desbordes et al. 2015, p.357).

According to Sahdra et al. (2010), nonattachment is positively related to mindfulness but empirically distinguishable from it and can also be enhanced by means of meditation. Coherently, higher levels of nonattachment were observed in individuals with meditative experience and significant correlations with nonattachment and contemplative practice/ experience were also reported (Sahdra et al. 2010). However, nonattachment is not developed by mindfulness or meditation alone since it increases throughout life along with lessons learned from life-challenging experiences such as the death of loved ones, other personal losses, or other significant circumstances (e.g., parenting), acting as a protective agent against suffering and as a promoter of a healthy psychological maturation (Sahdra et al. 2010). In common with other psychological qualities, including mindfulness, nonattachment can be observed on a continuum, being present to a certain extent in subjects with or without meditative experience (Sahdra et al. 2010).

The assessment of nonattachment is relatively new to science. Although McIntosh and Martin (1992) developed the Linking Inventory in the early 1990s for measuring nonattachment, understood as the extent to which respondents believe their happiness is independent of obtaining positive outcomes, it was not until the appearance of the work by Sahdra et al. (2010) that a specific psychological measure of the Buddhist conceptualization of nonattachment was proposed. In their study, Sahdra et al. (2010) developed the Nonattachment Scale (NAS) by means of exhaustive analysis and operationalization of several informative sources (classical Buddhist texts, contemporary Buddhist writings, etc.). The psychometric properties of the NAS were studied in USA student and adult samples to determine its factorial structure, internal consistency, temporal stability, and construct validity. Three hundred eighty-two undergraduates at a California university completed a 72-item preliminary version of the NAS as part of an online survey. In addition, 511 USA adults completed the 72-item scale in a separate, shorter online survey. The exploratory factor analysis (EFA) for the student data yielded a single factor that explained $28.6 \%$ of the item variance. A total of 32 items loaded above .40 on the factor. Using only these 32 items, they then conducted another EFA using the adult calibration sample data, finding that all but 2 of the 32 items loaded above .4 on a single factor. After deleting these two lower loading items, the remaining 30 items ( 3 of which were reverse-scored) explained $35.2 \%$ of total variance and demonstrated excellent internal consistency (.94). They also carried out a confirmatory factor analysis (CFA) using an adult validation sample, formulating 10 random parcels of the 30 -item scale. The CFA yielded a good fit to a singlefactor model. High correlations with mindfulness measures, meditation practice, and mental health variables were also reported in the validation of the original version of the NAS indicating that this measure seems to be valid and reliable in its English version. The authors acknowledged that their findings were limited in generalizability to USA college students and adults, and extensions of it should include testing the validity of the NAS in other cultures.

The main aim of the present study was to evaluate the dimensionality, internal consistency, and construct validity of the Spanish version of the NAS in a large sample of individuals with and without meditative experience. Specifically, we aimed at evaluating the relationship between the NAS and mindfulness facets and decentering, as well as the additional utility 
(beyond mindfulness and decentering instruments) of using NAS for predicting three mental health related indexes: psychopathology, emotional dysregulation, and resilience. Finally, this work also explores an important omission in the NAS literature by comparing NAS scores in three samples relevant to meditation research. Specifically, we analyzed the validity of the NAS for discriminating between meditators, nonmeditators, and patients with borderline personality disorder (BPD).

\section{Method}

\section{Participants}

The initial study sample consisted 921 Spanish individuals (67\% women) who were invited to voluntarily participate in the present study without remuneration. The subsample of nonpsychiatric volunteers was composed of 690 adult subjects (65\% women). Of these, 625 had complete NAS data. More than half of the participants $(n=355)$ reported some kind of previous meditative experience (mean lifetime of meditative practice $=7.12$ years) while 270 reported no experience at all. As described elsewhere (Soler et al. 2014a), the sample was recruited from the Autonomous University of Barcelona (Nursing and Psychology schools) and also by means of an internet-based commercial system (www.surveymonkey.com; Portland, OR, USA). A link to this website was posted on several Spanish scientific research and clinical portals of mindfulness and meditation. Additionally, this link was sent to several mindfulness associations, sanghas, and Zen monasteries and to a nonmeditative convenience sample. Finally, a clinical sample of 39 patients diagnosed with BPD from the Hospital de la Santa Creu i Sant Pau (Barcelona, Spain) was also included in this study in order to provide evidence on the utility of the NAS scale in clinical settings. BPD criteria were assessed by means of the Revised Diagnostic Interview for Borderlines (DIB-R; Spanish version by Barrachina et al. 2004).

Demographics of all participants and clinical data of the BPD group are displayed in Table 1.

\section{Procedure}

Permission to translate the NAS to Spanish was obtained from the original author (i.e., B.K. Sahdra). The NAS was translated from English to Spanish by two native bilingual English-Spanish speakers and then back-translated to obtain approval from the original authors. Any discrepancies between the Spanish and English versions were resolved by agreement. The online or paper-and-pencil battery of measures was completed by the nonclinical sample $(n=625)$, and all participants in the BPD sample $(n=39)$ filled out the paper-and-pencil version of all questionnaires during an appointment at the hospital's facilities.

\section{Measures}

The Nonattachment Scale (Sahdra et al. 2010) is a 30-item measure scored on a 6 -point scale ranging from $1=$ disagree strongly to $6=$ agree strongly and designed to measure nonattachment (e.g., "I can let go of regrets and feelings of dissatisfaction about the past," "When pleasant experiences end, I am fine moving on to what comes next"). The NAS was empirically derived from depuration of a pool of 135 items that had been obtained following careful revision of classical and contemporary Buddhist texts about nonattachment with the tuition and revision of several contemporary Buddhist teachers and scholars of known reputation in the field of meditation (and mindfulness) that were representative from the three major traditions in Buddhism (i.e., Theravadan, Zen, and Indo-Tibetan). Scores for the three negatively worded items of the NAS $(4,13$, and 24) are reversed, so that higher scores indicate more nonattachment. The NAS is unifactorial and shows excellent internal consistency (Cronbach alpha $=.94)$. Recently, an abridged (7-item) version of the original 30-item Nonattachment Scale was published (Sahdra et al. 2015); NAS-7 showed satisfactory internal consistency $(\alpha=.82)$.

The Five-Facet Mindfulness Questionnaire (FFMQ; Aguado et al. 2015; Baer et al. 2006; Baer et al. 2008; Cebolla et al. 2012) is a 39-item instrument rated on a fivepoint Likert scale $(1=$ never or very rarely true to $5=$ very often or always true), designed to evaluate mindfulness in a comprehensive way by assessing five key aspects of this construct: observing (e.g., "When I'm walking, I deliberately notice the sensations of my body moving"), describing (e.g., "I'm good at finding words to describe my feelings"), acting with awareness (e.g., "I find myself doing things without paying attention"), nonjudging of inner experience (e.g., "I criticize myself for having irrational or inappropriate emotions"), and nonreactivity to inner experience (e.g., "I perceive my feelings and emotions without having to react to them"). The Spanish version presented adequate Cronbach's alphas ranging from .80 to .91 (Cebolla et al. 2012).

The Experiences Questionnaire (EQ; Fresco et al. 2007a; Soler et al. 2014a) is an 11-item measure to assess decentering understood as the capacity to observe one's thoughts and feelings as temporary and objective events of the mind (e.g., "I can separate myself from my thoughts and feelings"). Items are rated on a five-point Likert scale (ranging from 1, never or very rarely true, to 5 , very often or always true) with higher scores reflecting greater decentering. The Spanish version of the EQ shows high internal consistency (Cronbach's $\alpha=.89$ ).

The Depression Anxiety Stress Scale, short form (DASS21; Lovibond and Lovibond 1995; Bados et al. 2005) is a 21item instrument with three sets of items to assess depressive symptomatology (e.g., "life is meaningless"), anxiety (e.g., "breathing problem"), and stress (e.g., "getting agitated") on a four-point scale assessing the severity/frequency of 
Table 1 Participant characteristics for the three samples (meditators, nonmeditators, and BPD patients)

\begin{tabular}{|c|c|c|c|}
\hline Characteristics & Meditators $(n=355)$ & Non-meditators $(n=270)$ & $\mathrm{BPD}(n=39)$ \\
\hline Gender (female): $n(\%)$ & $200(56.98)$ & $185(71.70)$ & $35(89.74)$ \\
\hline Age (years): M (SD) & $44.01(10.47)$ & $37.90(10.83)$ & $31.69(7.13)$ \\
\hline Years of education: M (SD) & $16.28(2.92)$ & $16.17(2.81)$ & $12.79(.41)$ \\
\hline Years of meditation experience (M, SD) & $7.24(8.05)$ & NA & NA \\
\hline Weekly hours of meditation (M, SD) & $2.15(2.18)$ & NA & NA \\
\hline Study measures & & $\mathrm{M}(\mathrm{SD})$ & \\
\hline NAS (1-6) & $4.92(.71)$ & $4.44(.81)$ & $3.02(.92)$ \\
\hline NAS-7 (1-6) & $5.11(.77)$ & $4.63(.93)$ & $3.10(.96)$ \\
\hline \multicolumn{4}{|l|}{ FFMQ } \\
\hline Observing (8-40) & $30.47(4.74)$ & $25.61(5.48)$ & $25.90(6.18)$ \\
\hline Describing $(8-40)$ & $30.52(5.32)$ & $29.28(6.00)$ & $24.23(6.18)$ \\
\hline Acting with awareness $(8-40)$ & $27.41(5.20)$ & $26.01(5.70)$ & $19.39(7.41)$ \\
\hline Nonjudging $(8-40)$ & $30.71(6.50)$ & $27.78(6.77)$ & $17.92(5.94)$ \\
\hline Nonreactivity (7-35) & $24.90(4.15)$ & $21.19(4.49)$ & $14.69(5.41)$ \\
\hline Average total score (1-5) & $3.69(.49)$ & $3.33(.48)$ & $2.63(.55)$ \\
\hline EQ (11-55) & $41.15(6.08)$ & $36.74(6.42)$ & $25.45(7.50)$ \\
\hline \multicolumn{4}{|l|}{ DASS-21 } \\
\hline Depression $(0-42)$ & $20.04(7.75)$ & $22.23(8.68)$ & $23.49(12.55)$ \\
\hline Anxiety $(0-42)$ & $19.09(6.47)$ & $20.61(7.35)$ & $20.56(11.59)$ \\
\hline Stress $(0-42)$ & $24.47(7.14)$ & $27.12(8.16)$ & $26.10(10.05)$ \\
\hline CD-RISC-10 (0-40) & $30.60(6.28)$ & $28.74(7.14)$ & $15.41(9.99)$ \\
\hline \multicolumn{4}{|l|}{ DERS } \\
\hline Non-acceptance (7-35) & $11.01(5.32)$ & $13.52(6.53)$ & $23.89(8.02)$ \\
\hline Interference (4-20) & $8.24(3.17)$ & $9.47(3.89)$ & $16.55(4.00)$ \\
\hline Non-control (9-45) & $13.06(5.66)$ & $15.15(7.08)$ & $34.35(8.26)$ \\
\hline Non-awareness (4-20) & $8.02(2.94)$ & $9.50(3.51)$ & $9.87(3.84)$ \\
\hline Confusion (4-20) & $6.31(2.40)$ & $7.23(2.98)$ & $11.21(4.33)$ \\
\hline DIB-R (0-10) & NA & NA & $7.89(1.31)$ \\
\hline
\end{tabular}

Total score ranges for each scale/subscales are represented between brackets

$C D$-RISC Connor-Davidson Resilience Scale-10, DASS-21 Depression Anxiety and Stress Scale, DIB-R Revised Diagnostic Interview for Borderlines, EQ Experiences Questionnaire, FFMQ Five Facet Mindfulness Questionnaire, NAS Nonattachment Scale, NA not available symptoms over the previous week. Cronbach's alpha values of the Spanish version of DASS-21 are high (.84 for depression, .80 for anxiety, and .82 for stress subscales).

The Connor-Davidson Resilience Scale-10 (CD-RISC-10; Connor and Davidson 2003; Notario-Pacheco et al. 2011) is a 10 -item self-administered measure designed to assess resilience (e.g., "I can deal with whatever comes"), a protective factor against mental problems and positively related to adaptive coping. Items from the CD-RISC-10 are formulated on a five-point rating (from $0=$ never to $4=$ almost always), and there is a total score ranging from 0 to 40 , with higher scores indicating greater levels of resilience; Cronbach's alpha of the Spanish version of the CD-RISC-10 is high $(\alpha=.85)$.

The Difficulties in Emotion Regulation Scale (DERS; Gratz and Roemer, 2004; Hervás and Jódar 2008) is a 28-item selfreport scale; its Spanish adaption has good psychometric properties (total $\alpha=.93$ ), which assesses maladaptive emotion regulation strategies across five areas: nonacceptance of emotions (e.g., "When I'm upset, I become angry with myself for feeling that way"), lack of emotional control (e.g., "When I'm upset, I lose control over my behavior."), lack of emotional awareness (e.g., "When I'm upset, I acknowledge my emotions"), interference in goal-directed behavior, and confusion (e.g., "When I'm upset, I have difficulty concentrating").

The meditative practice was assessed by means of ad hoc questions asking about experience in meditation (total years of personal practice), frequency of practice (times per week), and mean duration of a typical meditative practice session.

\section{Data Analyses}

Sociodemographic data were analyzed with descriptive statistics of mean, standard deviation (SD), and range. All data was analyzed with SPSS v22.0 and the Mplus v7.2. 
The sample was randomly split into two halves for the purpose of cross-sample validation, allowing for EFA (with the principal-axis method of estimation) with one half and CFA with the other ( $n=308$ and $n=317$, respectively).

Firstly, we made use of NAS scores for an EFA followed by varimax rotation. To explore the suitability of the data for factor analysis, the Kaiser-Meyer-Olkin (KMO) measure of sampling adequacy was computed. KMO scores $\geq .70$ are considered adequate. The Bartlett's test of Sphericity was also performed to examine the extent to which the correlation matrices departed from orthogonality. The following set of rules helped to determine the optimal number of components to retain (Tabachnick and Fidell 2007): Kaiser's criterion (components with eigenvalues $>1.0$ ), the Cattell scree test (inspection of a plot of the eigenvalues for breaks or discontinuities), and item loadings (an item forms part of a component if its factor loading on that factor is $\geq .32$ ).

Secondly, we tested the fit of the one-factor model by Sahdra et al. (2010), with all NAS items loading on one latent factor (Model 1). Unlike Sahdra et al. (2010), we did not make item parcels because this methodological approach spuriously increases model fit (Bandalos and Finney 2001). In addition, we tested a single factor model with method effects (model 2 as respecification of model 1) that incorporated correlated error terms on the negatively phrased items. A common outcome in the factor analysis of psychological instruments composed of positively and negatively worded items is to obtain an inadequate fit for unidimensional models because positively phrased items are prone to load on one factor and negatively phrased items on another (Woods 2006). The robust maximum likelihood (MLR) was applied to test the fit of the two factor models. The following indices were examined to evaluate model fit (Schumacher and Lomax 2010): chi-square (a nonsignificant estimate reflects good fit), the Tucker-Lewis index ( $\mathrm{TLI} \geq .90$ ), the comparative fit index (CFI $\geq .90$ ), and the root means square error of approximation (RMSEA $\leq .08$ ). Full Information Maximum Likelihood was used to account for missing values.

The scale's construct validity was studied by means of Pearson's correlations between NAS and mindfulness (FFMQ facets), decentering (EQ), resilience (CD-RISC-10), difficulties in emotional regulation (DERS), and clinical symptoms (i.e., depression, anxiety, and stress, DASS-21). Correlational analyses were performed separately in the meditative and nonmeditative samples. In order to better determine whether EQ scores presented higher overlapping with NAS scores than other mindfulness-related variables (i.e., FFMQ subscales), Steiger's Z tests (Steiger 1980) for dependent correlations were performed. Significant differences between NAS-EQ correlation and other NAS-FFMQ subscale correlations in both meditative and nonmeditative samples were explored. To assess whether the NAS is sensitive to individual differences in the practice of meditation, correlational analyses between NAS scores and practice-related variables (i.e., years of meditation practice, weekly hours of meditation, and number of days' practice per week) were also calculated. Given that older meditators may have more years of practice, an ulterior partial correlation between the NAS and years of meditation practice controlling by age was also performed. To examine the known-group construct validity, meditative, nonmeditative, and BPD samples were compared on NAS scores by means of an analysis of variance (ANOVA). Additionally, to determine whether the NAS presented similar known-group construct validity to other related measures, the FFMQ average total score was calculated and the effect sizes of the between-group differences regarding the NAS, FFMQ average total score, and the EQ were compared.

Finally, four hierarchical multiple regression analyses were performed to examine whether nonattachment was a significant predictor of clinical symptoms (depression, anxiety, and stress subscales from DASS-21) and resilience (CD-RISC$10)$. These analyses were performed controlling for sociodemographic variables and other mindfulness-related capacities (FFMQ and EQ). Specifically, due to the content overlap between the constructs of nonattachment and decentering, we wanted to explore whether one of these variables (i.e., nonattachment or decentering) was a stronger predictor of psychopathological symptoms. Sociodemographic variables were entered in the first step, followed by FFMQ scores and EQ scores in the second and third step, respectively. Finally, NAS scores were entered in step 4. Values regarding years of meditation practice and weekly hours of meditation variables in the nonmeditative sample were considered equal to zero in these last analyses.

\section{Results}

\section{Exploratory Factor Analysis}

The KMO yielded a coefficient of .95 , reflecting excellent sampling adequacy. The Bartlett's test of sphericity was $4980.38(p<.0001)$, indicating that the correlation matrix was suitable for factor analysis. Six components emerged in the EFA ( $n=308$ after listwise deletion) with eigenvalues $>1.0$. The first component explained $42.3 \%$ of the variance whereas the other five components explained 4.9, 4.4, 3.9, 3.5, and $3.4 \%$ of the variance, respectively (eigenvalues of the six components were $12.68,1.47,1.33,1.17,1.06$, and 1.01 , respectively). Considering that the criterion of eigenvalues greater than 1.0 can lead to overestimating the number of meaningful components, we decided to examine the scree plot and the pattern of factor loadings, which suggested that one component was sufficient to capture the essence of the Spanish NAS. A second EFA was performed, specifying that only one component should be extracted. The second EFA 
yielded a one-factor solution (accounting for $42 \%$ of total variance), with all items loading significantly on this factor $(\lambda \geq .32)$. Factor loadings for the one-factor model are presented in Table 2.

\section{Confirmatory Factor Analysis}

The $\chi^{2}$ value for both models was significant (model $1=1016.36$; model $2=1011.38 ; p<.001$ ). The other fit indices of model 1 did not indicate adequate fit to the data $(\mathrm{CFI}=.83 ; \mathrm{TLI}=.82)$ with the exception of the RMSEA that was .069 (90\% CI .064-.074). The inclusion of correlated residuals in model 2 did not improve model fit $(\mathrm{CFI}=.83$; $\mathrm{TLI}=.82$; RMSEA $=.069,90 \%$ CI $.064-.074)$. In addition, the three correlated residuals were not statistically significant $\left(\theta_{4,13}=.02 ; \theta_{4,24}=.02 ; \theta_{13,24}=.13\right)$. Therefore, model 1 should be retained because of parsimony considerations. Standardized factor loadings were all statistically significant $(p<.01)$ in model 1, ranging from a minimum of .38 (item 28) to a maximum of .77 (item 3 ). Instead of performing a search for the sources of misfit in modification indices, we decided to analyze whether model 1 provided good fit to the data for the NAS-7 using MLR as estimation method as suggested by one anonymous reviewer. Although the chi-square was significant

Table 2 Means (M), standard deviations (SD), and factor loadings $(\lambda)$ for all NAS items

\begin{tabular}{|c|c|c|c|c|}
\hline \multirow[t]{2}{*}{ NAS items } & \multicolumn{2}{|c|}{$\mathrm{EFA}(n=308)$} & \multicolumn{2}{|c|}{ CFA $(n=317)$} \\
\hline & $\mathrm{M}(\mathrm{SD})$ & $\lambda$ & $\mathrm{M}(\mathrm{SD})$ & $\lambda$ NAS/NAS-7 \\
\hline 1. I can accept the flow of events in my life without hanging onto them or pushing them away. & $4.22(1.37)$ & .64 & $4.43(1.29)$ & .56 \\
\hline 2. I can let go of regrets and feelings of dissatisfaction about the past. & $4.50(1.42)$ & .70 & $4.58(1.37)$ & $.70 / .61$ \\
\hline 3. I find I can be calm and/or happy even if things are not going my way. & $4.27(1.33)$ & .77 & $4.44(1.24)$ & .77 \\
\hline 4. I have a hard time appreciating others' successes when they outperform me. & $4.62(1.48)$ & .46 & $4.63(1.40)$ & .73 \\
\hline $\begin{array}{l}\text { 5. I can remain open to what life offers me regardless of whether it seems } \\
\text { desirable or undesirable at a particular time. }\end{array}$ & $4.62(1.20)$ & 69 & $4.58(1.25)$ & .64 \\
\hline 6. I can enjoy pleasant experiences without needing them to last forever. & $5.21(0.99)$ & .66 & $5.13(1.16)$ & $.74 / .74$ \\
\hline $\begin{array}{l}\text { 7. I view the problems that enter my life as things/issues to work on rather } \\
\text { than reasons for becoming disheartened or demoralized. }\end{array}$ & $4.90(1.29)$ & .73 & $4.97(1.25)$ & $.57 / .79$ \\
\hline 8. I can enjoy my possessions without being upset when they are damaged or destroyed. & $4.69(1.23)$ & .66 & $4.72(1.26)$ & .48 \\
\hline 9. The amount of money I have is not important to my sense of who I am. & $5.06(1.30)$ & .54 & $5.13(1.27)$ & .69 \\
\hline 10. I do not go out of my way to cover up or deny my negative qualities or mistakes. & $4.78(1.13)$ & .61 & $4.79(1.18)$ & .65 \\
\hline 11. I accept my flaws. & $4.70(1.18)$ & .76 & $4.77(1.10)$ & .69 \\
\hline 12. I can enjoy my family and friends without feeling I need to hang on to them. & $4.86(1.21)$ & .72 & $4.88(1.30)$ & $.76 / .77$ \\
\hline 13. If things aren't turning out the way I want, I get upset. & $3.93(1.46)$ & .45 & $3.96(1.41)$ & .56 \\
\hline 14. I can enjoy the pleasures of life without feeling sad or frustrated when they end. & $4.92(1.16)$ & .70 & $4.99(1.10)$ & .64 \\
\hline 15. I can take joy in others' achievements without feeling envious. & $5.03(1.13)$ & .50 & $5.05(1.07)$ & $.66 / .66$ \\
\hline 16. I find I can be happy almost regardless of what is going on in my life. & $3.91(1.38)$ & .64 & $3.84(1.43)$ & .62 \\
\hline 17. Instead of avoiding or denying life's difficulties, I face up to them. & $4.88(1.06)$ & 67 & $4.88(1.16)$ & .71 \\
\hline 18. I am open to reflecting on my past mistakes and failings. & $5.25(0.95)$ & .62 & $5.28(0.94)$ & .70 \\
\hline 19. I do not get "hung up" on wanting an "ideal" or "perfect" life. & $4.87(1.23)$ & .68 & $4.86(1.23)$ & $.74 / .77$ \\
\hline 20. I am comfortable being an ordinary, less than perfect human being. & $4.87(1.25)$ & 67 & $4.92(1.24)$ & .72 \\
\hline $\begin{array}{l}\text { 21. I can remain open to thoughts and feelings that come into my mind, } \\
\text { even if they are negative or painful. }\end{array}$ & $4.81(1.05)$ & .71 & $4.87(1.09)$ & .71 \\
\hline $\begin{array}{l}\text { 22. I can see my own problems and shortcomings without trying to blame } \\
\text { them on someone or something outside myself. }\end{array}$ & $5.02(1.09)$ & .76 & $5.12(1.05)$ & .53 \\
\hline 23. When pleasant experiences end, I am fine moving on to what comes next. & $4.87(1.10)$ & .62 & $4.91(1.08)$ & $.62 / .56$ \\
\hline 24. I am often preoccupied by threats or fears. & $4.27(1.47)$ & .33 & $4.23(1.55)$ & .57 \\
\hline 25. I am not possessive of the people I love. & $4.64(1.32)$ & .54 & $4.73(1.30)$ & .74 \\
\hline 26. I do not have to hang on to the people I love at all costs; I can let them go if they wish to go. & $4.71(1.32)$ & .60 & $4.81(1.23)$ & .64 \\
\hline 27. I do not feel I need to escape or avoid bad experiences in my life. & $4.26(1.32)$ & .62 & $4.38(1.32)$ & .55 \\
\hline 28. I can admit my shortcomings without shame or embarrassment. & $4.65(1.19)$ & .65 & $4.80(1.17)$ & .38 \\
\hline $\begin{array}{l}\text { 29. I experience and acknowledge grief following significant losses, but do } \\
\text { not become overwhelmed, devastated, or incapable of meeting life's other demands. }\end{array}$ & $4.64(1.28)$ & .62 & $4.80(1.23)$ & .38 \\
\hline 30. I am not possessive of the things I own. & $4.61(1.23)$ & .55 & $4.70(1.17)$ & .45 \\
\hline
\end{tabular}

Items in italics are the items included in the short version of the Nonattachment Scale or NAS-7 
$\left(\chi^{2}=46.897 ; p<.001\right)$ and the RMSEA of $.086(90 \% \mathrm{CI}$ $.060-.114)$ fell above the .080 cutoff, the other fit indices indicated adequate fit to the data $(\mathrm{CFI}=.94 ; \mathrm{TLI}=.91)$. Factor loadings for the NAS (30 items) and NAS-7 are shown in Table 2.

\section{Internal Consistency}

The Spanish NAS showed excellent internal consistency in the total sample $(\alpha=.949)$, in meditators $(\alpha=.947)$ and in nonmeditators $(\alpha=.943)$. The elimination of the inversescored items $(4,13$, and 24$)$ did not significantly increase the reliability of the scale. The NAS-7 showed satisfactory internal consistency in meditators $(\alpha=.850)$, in nonmeditators $(\alpha=.832)$, and in the total sample $(\alpha=.850)$.

\section{Relationship Between Nonattachment and Mindfulness-Related Constructs, Psychopathological Symptoms, Emotion Regulation, and Resilience}

All correlations between NAS and other variables were in the expected direction $(n=351$ and 258 after listwise deletion in the correlational analyses with meditators and nonmeditators, respectively). Correlational statistics are detailed in Tables 3 and 4.
Nonmeditative group: Correlations between NAS and mindfulness facets were significant and weak to moderate, from $r=.217$ (FFMQ-observing) to .532 (FFMQnonreactivity). The NAS and the EQ were strongly associated $(r=.733)$ (see Table 2). Correlations between nonattachment and difficulties in regulating emotions (DERS subscales) were also weak to moderate [from $r=-.250$ (nonawareness) to $r=-.615$ (noncontrol)]. The NAS was also moderately associated with DASS-21 subscales [from $r=-.465$ (anxiety) to $r=-.532$ (depression)]. Considering Steiger's Z tests (all $p<.0001$ ), for both groups (i.e., meditative and nonmeditative), NAS scores presented a significantly stronger correlation with EQ scores than with FFMQ facets. For more detailed information, see Table 3.

Meditative group: The magnitude of the correlations between the NAS and the FFMQ subscales (mindfulness facets) and the EQ (decentering) were moderate to high (all $r>.447$ ) and highly significant (all $p<.0001$ ), especially with decentering $(r=.745)$ and two FFMQ facets: nonreactivity $(r=.614)$ and nonjudge $(r=.540)$. See Table 3 for more details. Correlations between NAS and CD-RISC-10 (resilience) were also highly significant and with moderate-to-strong effect size $(r=.726)$. The correlations between NAS scores and DERS subscales were negative, with a significant $p$ value (all $p<.001$ ) and moderate in magnitude [from $r=-.374$
Table 3 Convergent validity: Pearson's correlations between NAS, NAS-7, EQ, and FFMQ subscales

\begin{tabular}{|c|c|c|c|c|c|c|c|}
\hline Meditative group & NAS-7 & EQ & Observe & Describe & Awareness & Nonjudge & Nonreact \\
\hline NAS & $.929 *$ & $.745^{*}$ & $.510^{*}$ & $.447^{*}$ & $.535 *$ & $.540 *$ & $.614 *$ \\
\hline NAS-7 & & $.665^{*}$ & $.482 *$ & $.395^{*}$ & $.471 *$ & $.515^{*}$ & $.557 *$ \\
\hline EQ & & & $.524 *$ & $.493 *$ & $.613 *$ & $.587 *$ & $.582 *$ \\
\hline FFMQ-observe & & & & $.397 *$ & $.395 *$ & $.265^{*}$ & $.615^{*}$ \\
\hline FFMQ-describe & & & & & $.368^{*}$ & $.385^{*}$ & $.459 *$ \\
\hline FFMQ-awareness & & & & & & $.460 *$ & $.437^{*}$ \\
\hline FFMQ-nonjudge & & & & & & & $.469 *$ \\
\hline \multicolumn{8}{|c|}{ Non-meditative group } \\
\hline NAS & $.926 *$ & $.733 *$ & $.217 * *$ & $.358 *$ & $.464 *$ & $.526^{*}$ & $.532 *$ \\
\hline NAS-7 & & $.684^{*}$ & $.279 *$ & $.327^{*}$ & $.407 *$ & $.467 *$ & $.502 *$ \\
\hline EQ & & & $.324 *$ & $.455^{*}$ & $.499 *$ & $.475^{*}$ & $.622 *$ \\
\hline FFMQ-observe & & & & $.237 * *$ & .067 & -.012 & $.325 *$ \\
\hline FFMQ-describe & & & & & $.347^{*}$ & $.329 *$ & $.378 *$ \\
\hline FFMQ-awareness & & & & & & $.463 *$ & $.379 *$ \\
\hline FFMQ-nonjudge & & & & & & & $.380 *$ \\
\hline
\end{tabular}

Observe, describe, awareness, nonjudge, and nonreactivity correspond to the Five Facet Mindfulness Questionnaire (FFMQ) subscales. Steiger's Z test for significance of the difference between dependent correlations showed that the correlation between NAS and EQ was significantly greater than the correlations between NAS and FFMQ facets, in meditators $\left[r_{N A S-E Q}\right.$ vs. $r_{N A S-O b s e r v e}: Z=6506, p<.0001$; vs. $\mathrm{r}_{\text {NAS-Describe }}: Z=7796, p$ $<.0001$; vs. $\mathrm{r}_{\mathrm{NAS}-\text { Awareness }}: Z=6440, p<.0001$; vs. $\mathrm{r}_{\text {NAS-Nonjudge: }} Z=6126, p<.0001$; vs. $\mathrm{r}_{\text {NAS-Nonreact }}: Z=4571$, $p<.0001$ ] and in non-meditators [ $\mathrm{r}_{\text {NAS-EQ }}$ vs. $\mathrm{r}_{\text {NAS-Observe }}: Z=9531, p<.0001$; vs. $\mathrm{r}_{\text {NAS-Describe }}: Z=7948, p$ $<.0001$; vs. $\mathrm{r}_{\text {NAS-Awareness }}: Z=6159, p<.0001$; vs. $\mathrm{r}_{\text {NAS-Nonjudge }}: Z=4797, p<.0001$; vs. $\mathrm{r}_{\text {NAS-Nonreact }}: Z=$ $5375, p<.0001]$

NAS Nonattachment Scale, EQ Experiences Questionnaire

${ }^{*} p<.0001 ; * * p<.0005$ 
Table 4 Convergent validity: Pearson's correlations between NAS, NAS-7, EQ, and FFMQ facets with DERS subscales, DASS-21 subscales, and CD-RISC

\begin{tabular}{|c|c|c|c|c|c|c|c|c|c|}
\hline Meditative group & Nonacceptance & Interference & Noncontrol & Nonawareness & Confusion & DASS-D & DASS-A & DASS-S & CD-RISC \\
\hline NAS & $-.630^{*}$ & $-.545^{*}$ & $-.665^{*}$ & $-.374 *$ & $-.574 *$ & $-.605^{*}$ & $-.515^{*}$ & $-.617^{*}$ & $.726^{*}$ \\
\hline NAS-7 & $-.591^{*}$ & $-.505^{*}$ & $-.614^{*}$ & $-.308^{*}$ & $-.526^{*}$ & $-.571^{*}$ & $-.480^{*}$ & $-.574 *$ & $.639 *$ \\
\hline EQ & $-.622^{*}$ & $-.535^{*}$ & $-.623^{*}$ & $-.469^{*}$ & $-.583^{*}$ & $-.566^{*}$ & $-.510^{*}$ & $-.606^{*}$ & $.691^{*}$ \\
\hline FFMQ-observe & $-.358^{*}$ & $-.344 *$ & $-.391 *$ & $-.489 *$ & $-.345^{*}$ & $-.355^{*}$ & $-.291 *$ & $-.396^{*}$ & $.421^{*}$ \\
\hline FFMQ-describe & $-.455^{*}$ & $-.437 *$ & $-.457^{*}$ & $-.489^{*}$ & $-.670^{*}$ & $-.483 *$ & $-.434 *$ & $-.447 *$ & $.494 *$ \\
\hline FFMQ-awareness & $-.418^{*}$ & $-.417 *$ & $-.444^{*}$ & $-.363^{*}$ & $-.450^{*}$ & $-.408^{*}$ & $-.365^{*}$ & $-.509^{*}$ & $.510^{*}$ \\
\hline FFMQ-nonjudge & $-.681^{*}$ & $-.413^{*}$ & $-.541^{*}$ & $-.250^{*}$ & $-.500^{*}$ & $-.496^{*}$ & $-.447^{*}$ & $-.514^{*}$ & $.407 *$ \\
\hline FFMQ-nonreact & $-.544^{*}$ & $-.471^{*}$ & $-.584^{*}$ & $-.375^{*}$ & $-.515^{*}$ & $-.499 *$ & $-.482^{*}$ & $-.580^{*}$ & $.544 *$ \\
\hline DERS-nonacceptance & & $.660^{*}$ & $.798^{*}$ & $.221 *$ & $.645^{*}$ & $.702 *$ & $.679 *$ & $.658^{*}$ & $-.520^{*}$ \\
\hline DERS-interference & & & $.713^{*}$ & $.263^{*}$ & $.597 *$ & $.609 *$ & $.534^{*}$ & $.587^{*}$ & $-.510^{*}$ \\
\hline DERS-noncontrol & & & & $.230^{*}$ & $.677^{*}$ & $.726^{*}$ & $.689 *$ & $.698^{*}$ & $-.576^{*}$ \\
\hline DERS-nonawareness & & & & & $.477^{*}$ & $.305^{*}$ & $.261^{*}$ & $.327^{*}$ & $-.363^{*}$ \\
\hline DERS-confusion & & & & & & $.655^{*}$ & $.635^{*}$ & $.609 *$ & $-.569^{*}$ \\
\hline DASS-D & & & & & & & $.721^{*}$ & $.707^{*}$ & $-.587 *$ \\
\hline DASS-A & & & & & & & & $.752^{*}$ & $-.444 *$ \\
\hline DASS-S & & & & & & & & & $-.485^{*}$ \\
\hline \multicolumn{10}{|l|}{ Nonmeditative group } \\
\hline NAS & $-.472 *$ & $-.470^{*}$ & $-.615^{*}$ & $-.250^{*}$ & $-.456^{*}$ & $-.532 *$ & $-.465^{*}$ & $-.525^{*}$ & $.692 *$ \\
\hline NAS-7 & $-.403^{*}$ & $-.383^{*}$ & $-.555^{*}$ & $-.257^{*}$ & $-.435^{*}$ & $-.493^{*}$ & $-.421^{*}$ & $-.439^{*}$ & $.617^{*}$ \\
\hline EQ & $-.469^{*}$ & $-.461^{*}$ & $-.590^{*}$ & $-.404 *$ & $-.565^{*}$ & $-.520^{*}$ & $-.425^{*}$ & $-.499 *$ & $.738^{*}$ \\
\hline FFMQ-observe & -.006 & -.010 & -.110 & $-.491 *$ & $-.279 *$ & -.108 & -.015 & -.020 & $.183^{*}$ \\
\hline FFMQ-describe & $-.252 * *$ & $-.217 * *$ & $-.296^{*}$ & $-.481^{*}$ & $-.578^{*}$ & $-.351^{*}$ & $-.337 *$ & $-.230^{*}$ & $.490 *$ \\
\hline FFMQ-awareness & $-.419^{*}$ & $-.552 *$ & $-.512 *$ & -.116 & $-.394 *$ & $-.477^{*}$ & $-.460^{*}$ & $-486^{*}$ & $.421 *$ \\
\hline FFMQ-nonjudge & $-.616^{*}$ & $-.357^{*}$ & $-.557 *$ & $-.119 * * *$ & $-.426^{*}$ & $-.474 *$ & $-.514^{*}$ & $-.499 *$ & $.450^{*}$ \\
\hline FFMQ-nonreact & $-.409^{*}$ & $-.376^{*}$ & $-.516^{*}$ & $-.296^{*}$ & $-.445^{*}$ & $-.370^{*}$ & $-.359 *$ & $-.342 *$ & $.490 *$ \\
\hline DERS-nonacceptance & & $.568^{*}$ & $.695 *$ & .056 & $.471^{*}$ & $.539 *$ & $.547 *$ & $.540^{*}$ & $-.446^{*}$ \\
\hline DERS-interference & & & $.693^{*}$ & .067 & $.440^{*}$ & $.524 *$ & $.519 *$ & $.591^{*}$ & $-.482 *$ \\
\hline DERS-noncontrol & & & & .107 & $.565^{*}$ & $.616^{*}$ & $.649 *$ & $.682 *$ & $-.569^{*}$ \\
\hline DERS-nonawareness & & & & & $.524 *$ & $.216^{* *}$ & .116 & .070 & $-.328^{*}$ \\
\hline DERS-confusion & & & & & & $.602^{*}$ & $.476^{*}$ & $.468^{*}$ & $-.514^{*}$ \\
\hline DASS-D & & & & & & & $.681^{*}$ & $.646^{*}$ & $-.476^{*}$ \\
\hline DASS-A & & & & & & & & $.758^{*}$ & $-.424 *$ \\
\hline DASS-S & & & & & & & & & $-.446^{*}$ \\
\hline
\end{tabular}

Depression Anxiety and Stress Scale (DASS-21) subscales are also represented (i.e., DASS-D, DASS-A, and DASS-S, respectively)

$N A S$ Nonattachment Scale, EQ Experiences Questionnaire, CD-RISC-10 Connor-Davidson Resilience Scale-10, DERS Difficulties in Emotion Regulation Scale

${ }^{*} p<.0001 ; * * p .0005 ; * * * \leq .05$

(nonawareness) to -.665 (noncontrol)]. Correlations between NAS and DASS-21 subscales were of moderate effect size [from $r=-.515$ (DASS-anxiety) to -.617 (DASS-stress)]. See Table 4 for detailed information. As expected, the NAS7 showed high association with the NAS $(r=.93)$ and a similar pattern of correlations with the other instruments (see Tables 3 and 4).

Significant correlations were also shown between years of meditation practice and the NAS $(n=313 ; r=.138$, $p=.015)$ and between weekly hours of meditation and frequency of meditative practice (i.e., days per week) and the NAS $(n=343 ; r=.216, p<.0001$ and $n=351$; $r=.273, p<.0001$, respectively). Partial correlation controlling the effect of age on the relationship between the NAS and years of meditation practice indicated a trend towards significance in the specific relation between the NAS and years of meditation $(n=307 ; r=.105, p=.07)$. Significant correlations between the NAS and age $(n=$ 
$607 ; r=.251, p<.0001)$ and years of schooling $(n=609$; $r=.091, p=.021$ ) were also found. Men obtained higher NAS scores than women $[$ means $=4.77(.78)$ vs. 4.54 (.92), respectively; $t=-3.411, d f=531.793, p=.001]$.

\section{Unique Features of Nonattachment: the Added Value of the NAS as Predictor of Psychopathological Symptoms and Resilience}

These analyses provided a stringent test of the NAS' capacity to predict specific types of psychopathological symptoms after controlling for sociodemographic characteristics, mindfulness facets, and decentering. As mentioned previously, four hierarchical regression analyses were computed with depressive symptoms (DASS-21-D), anxious symptoms (DASS-21A), stress symptoms (DASS-21-S), and resilience (CD-RISC10) as the criterion variables. In each analysis gender, age, years of education, and meditation practice were entered as covariates on step 1, the FFMQ subscales were entered together on step 2, and then the EQ and the NAS were entered separately on steps 3 and 4 , respectively.

All four-regression analyses were highly significant, with the measures accounting for $46.8 \%$ of the DASS-21-D variance, $40.2 \%$ of the DASS-21-A variance, $47.8 \%$ of the variability in the DASS-21-S, and $61.6 \%$ of the CD-RISC10 variance. The results found in steps 3 and 4 are of particular interest to the present work. In each analysis, the NAS emerged as a significant unique predictor of depressive, anxiety, stress symptoms, and resilience, even after controlling for the influence of other important mindfulness-related constructs. The amount of additional variance explained by NAS in these variables was different in each case: $2.3 \%$ (DASS-21-D), $1.2 \%$ (DASS-21-A), $2.1 \%$ (DASS-21-S), and $5.7 \%$ (CD-RISC-10). Thus, nonattachment seems to play a greater role in predicting resilience and depression, than in predicting stress symptoms or anxious states. In addition to NAS scores, other variables were also significant predictors of each symptom scale. DASS-21-D variance was also predicted by age, years of schooling, three FFMQ subscales (i.e., describing, acting with awareness, and nonjudging), and EQ scores. DASS21-A was predicted by years of schooling and observing, describing, acting with awareness, and nonjudging subscales. DASS-21-S was also predicted by FFMQ-observing, acting with awareness, and nonjudging. Lastly, CD-RISC-10 scores were also predicted by age, observing, describing, and EQ. It is worth mentioning the differential role of the FFMQ-observing facet when predicting DASS-21-A, DASS-21-S, and CD-RISC-10 scores, since higher levels of the observe facet predicted higher levels of anxiety and stress $(\beta=.138$ and .099 , respectively) and lower levels of resilience $(\beta=-.090)$. See Table 5 for more detailed information.
Table 5 Summary of hierarchical regression analyses of depressive, anxious, and stress symptoms from DASS-21 and resilience measured with the CD-RISC-10

\begin{tabular}{|c|c|c|c|c|c|}
\hline Predictor variables & $\Delta R^{2}$ & $r$ & Beta & $\mathrm{sr}^{2}$ & $t$ value \\
\hline \multicolumn{6}{|c|}{ With DASS-21 Depression as Criterion } \\
\hline Step 1 & .071 & & & & \\
\hline Age & & -.029 & .107 & .01 & $3.060 *$ \\
\hline Sex & & -.030 & .030 & .00 & .891 \\
\hline Years of schooling & & -.192 & -.108 & .01 & $-3.330^{*}$ \\
\hline Years of meditation practice & & -.139 & -.009 & .00 & -.237 \\
\hline Weekly hours of meditation & & -.142 & -.010 & .00 & -.287 \\
\hline Step 2 & .335 & & & & \\
\hline FFMQ-observe & & -.233 & .078 & .00 & 1.940 \\
\hline FFMQ-describe & & -.418 & -.095 & .01 & $-2.492 *$ \\
\hline FFMQ-awareness & & -.493 & -.160 & .02 & $-4.035^{*}$ \\
\hline FFMQ-nonjudge & & -.519 & -.177 & .02 & $-4.313^{*}$ \\
\hline FFMQ-nonreact & & -.428 & .011 & .00 & .228 \\
\hline Step 3 & .038 & & & & \\
\hline EQ & & -.571 & -.202 & .01 & $-3.386^{*}$ \\
\hline Step 4 & .023 & & & & \\
\hline NAS & & -.579 & -.252 & .02 & $-4.896^{*}$ \\
\hline
\end{tabular}

Note: ${ }^{*} p<.05 . R=.68, R^{2}=.47, F(12,544)=39.870, p<.0001$

With DASS-21 anxiety as criterion

Step 1

Age

.067

Sex

Years of schooling

Years of meditation practice

Weekly hours of meditation

Step 2

FFMQ-observe

FFMQ-describe

FFMQ-awareness

FFMQ-nonjudge

FFMQ-nonreact

Step 3

EQ

$\begin{array}{llll}-.139 & -.035 & .00 & -.948\end{array}$

$\begin{array}{llll}-.104 & -.032 & .00 & -.901\end{array}$

$\begin{array}{llll}-.174 & -.089 & .01 & -2.590 *\end{array}$

$\begin{array}{llll}-.118 & .040 & .00 & 1.023\end{array}$

$\begin{array}{llll}-.125 & -.002 & .00 & -.044\end{array}$

311

$\begin{array}{llll}-.145 & .138 & .01 & 3.216^{*}\end{array}$

$\begin{array}{llll}-.392 & -.131 & .01 & -3.252^{*}\end{array}$

$\begin{array}{llll}-.460 & -.164 & .02 & -3.906 *\end{array}$

$\begin{array}{llll}-.515 & -.223 & .03 & -5.131^{*}\end{array}$

$\begin{array}{lllll}-.405 & -.056 & .00 & -1.083\end{array}$

Step 4

NAS

.012

$\begin{array}{llll}-.497 & -.097 & .00 & -1.531\end{array}$

.012

$\begin{array}{llll}-.505 & -.179 & .01 & -3.295 *\end{array}$

Note: ${ }^{*} p \leq .01 . R=.63, R^{2}=.40, F(12,544)=30.517, p<.0001$

With DASS-21 stress as criterion

Step 1

.077

Age

Sex

Years of schooling

Years of meditation practice

Weekly hours of meditation

Step 2

FFMQ-observe

FFMQ-describe

FFMQ-awareness

$$
\begin{array}{rrrrr}
.077 & & & & \\
& -.139 & .026 & .00 & .750 \\
& -.105 & -.006 & .00 & -.190 \\
& -.102 & -.027 & .00 & -.832 \\
& -.218 & -.044 & .00 & -1.199 \\
& -.184 & .002 & .00 & .060 \\
.348 & & & & \\
& -.236 & .099 & .01 & 2.466^{*} \\
& -.346 & -.008 & .00 & -.211 \\
& -.540 & -.219 & .03 & -5.582^{*}
\end{array}
$$


Table 5 (continued)

\begin{tabular}{|c|c|c|c|c|c|}
\hline Predictor variables & $\Delta R^{2}$ & $r$ & Beta & $\mathrm{sr}^{2}$ & $t$ value \\
\hline FFMQ-nonjudge & & -.538 & -.182 & .02 & $-4.472^{*}$ \\
\hline FFMQ-nonreact & & -.472 & -.050 & .00 & -1.030 \\
\hline Step 3 & .033 & & & & \\
\hline EQ & & -.590 & -.184 & .01 & $-3.107^{*}$ \\
\hline Step 4 & .021 & & & & \\
\hline NAS & & -.597 & -.238 & .02 & $-4.674^{*}$ \\
\hline \multicolumn{6}{|c|}{ Note: ${ }^{*} p<.05 . R=.69, R^{2}=.48, F(12,544)=41.580, p<.0001$} \\
\hline \multicolumn{6}{|c|}{ With CD-RISC-10 as criterion } \\
\hline Step 1 & .047 & & & & \\
\hline Age & & .052 & -.083 & .01 & $-2.762 *$ \\
\hline Sex & & .017 & -.046 & .00 & -1.625 \\
\hline Years of schooling & & .152 & .034 & .00 & 1.215 \\
\hline Years of meditation practice & & .119 & -.034 & .00 & -1.064 \\
\hline Weekly hours of meditation & & .125 & -.055 & .00 & -1.755 \\
\hline Step 2 & .391 & & & & \\
\hline FFMQ-observe & & .313 & -.090 & .00 & $-2.617^{*}$ \\
\hline FFMQ-describe & & .511 & .174 & .02 & $5.352 *$ \\
\hline FFMQ-awareness & & .488 & .061 & .00 & 1.806 \\
\hline FFMQ-nonjudge & & .447 & -.056 & .00 & -1.588 \\
\hline FFMQ-nonreact & & .514 & .032 & .00 & .776 \\
\hline Step 3 & .120 & & & & \\
\hline EQ & & .701 & .386 & .04 & $7.591 *$ \\
\hline Step 4 & .057 & & & & \\
\hline NAS & & 697 & .393 & .06 & $8.972 *$ \\
\hline
\end{tabular}

FFMQ Five Facet Mindfulness Questionnaire, EQ Experiences Questionnaire, NAS Nonattachment Scale, DASS-21 Depression Anxiety and Stress Scale, CD-RISC-10 Connor-Davidson Resilience Scale-10

${ }^{*} p<.01 . R=.78, R^{2}=.62, F(12,540)=72.180, p<.0001$

\section{Criterion-Related (Known-Group) Validity: Comparisons of Group Means on the NAS}

A highly significant between-group (i.e., meditative, nonmeditative, and BPD) difference in NAS scores was found in the one-way ANOVA $[F(2,654)=104.009, p$ $\left.<.0001, \eta^{2}=.24\right]$. The Bonferroni-corrected post hoc comparisons indicated that the BPD group reported significantly lower NAS scores $[$ mean $=3.02(.92)]$ compared with the nonmeditative group (mean $=4.44(.81), p<.0001, d=$ $-1.63)$ and, especially, compared with the meditative group (mean $=4.92(.71), p<.0001, d=-2.30)$. Furthermore, the nonmeditative group also showed lower NAS scores when compared with the meditation group $(p<.0001, d=-.62)$. Significant differences between groups were also found in the NAS-7 scores $[F(2,654)=102.322, p<.0001$, $\left.\eta^{2}=.24\right]$ : BPD vs. nonmeditative $(p<.0001, d=-1.62)$, BPD vs. meditative $(p<.0001, d=-2.30)$, and nonmeditative vs. meditative $(p<.0001, d=-.56)$.
Significant between-group differences in FFMQ average total scores were reported $[F(2,644)=103.249, p<.0001$, $\left.\eta^{2}=.24\right]$ : BPD vs. nonmeditative $(p<.0001, d=-1.35)$, BPD vs. meditative $(p<.0001, d=-2.04)$, and nonmeditative vs. meditative $(p<.0001, d=-.75)$. Significant differences among groups regarding EQ scores were also observed $[F(2,660)=123.285, p<.0001$, $\left.\eta^{2}=.27\right]$ : BPD vs. nonmeditative $(p<.0001, d=-1.62)$, BPD vs. meditative $(p<.0001, d=-2.30)$, and nonmeditative vs. meditative $(p<.0001, d=-.71)$. Please see Table 1 for means and standard deviations of the NAS7, FFMQ, and EQ. Effect sizes for the differences among groups regarding NAS scores were comparable and consistent with those effect sizes observed for the FFMQ average total score and the EQ. All these effect sizes were moderate according to Ferguson (2009).

\section{Discussion}

The Spanish version of the NAS shows a unifactorial structure and similar psychometric properties to the original version of the instrument (Sahdra et al. 2010). The NAS has an excellent internal reliability and high convergence with mindfulness facets and with decentering, especially in the meditative group. Interestingly, the NAS showed significantly higher correlation with EQ when such correlation is compared with all NAS-FFMQ facet correlations in both meditative and nonmeditative groups, suggesting a higher overlap between nonattachment and decentering constructs. Our results also indicate that high nonattachment may be a protective factor against emotional distress, since significant and positive associations were found between NAS scores and resilience. Conversely, strong negative correlations were found between NAS scores and measures of emotional dysregulation and psychopathology (depression, anxiety, and stress). Moreover, the construct of nonattachment seems to incorporate something beyond mindfulness and decentering when predicting psychopathology and, especially, when predicting resilience, as suggested by hierarchical linear regression analyses. Finally, in line with the aforementioned findings, the group with BPD diagnosis presented the lowest levels of nonattachment when compared to the meditator and the nonmeditator groups, emphasizing that a lack of nonattachment would be allied with mental health disturbances. An enhancing effect of meditation on levels of nonattachment was also suggested by our findings since higher NAS scores were found in the meditative group and positive correlations between the NAS and the practice of meditation were also observed.

The results of the EFA and CFA bring out a one-component structure of the NAS Spanish version, which is congruent with the factor analyses conducted in the original validation of the scale (Sahdra et al. 2010). Unsatisfactory fit indices to the one- 
factor model were obtained in the CFA of the Spanish version of the NAS. In comparison to the validation of the original scale, this model obtained a worse fit. This discrepancy may be due to different approaches when performing the CFA, since Sahdra et al. (2010) used an item-parceling approach and we used an individual-item (without parcels) approach. The use of item parceling has been a source of intense debate (Bandalos and Finney 2001). Although NAS-7 had a notably better model fit than the original 30-item NAS, we have to bear in mind that "it is important to validate the short form in the form it will be used, rather than by extracting its items or observations from the fulllength assessment" (Smith et al. 2000, p. 110). Compared to the 30-item NAS, the abridged form showed lower internal consistency and similar convergence with other instruments measuring mindfulness, decentering, resilience, emotional dysregulation, and psychopathology. In our opinion, the NAS-7 might be a better option than the 30-item NAS in busy settings with scant time for evaluations or as part of large batteries of instruments.

The internal consistency of the Spanish version of the NAS was excellent in both meditative and nonmeditative samples, suggesting that this is a reliable measure independently of the meditative background of the sample. The NAS highly correlated with mindfulness facets, especially in the sample with meditative experience. Lower convergence between mindfulness-related variables in nonmeditative samples has also been reported before (e.g., de Bruin et al. 2012), with observing the most problematic facet because of its erratic association with other mindfulness constructs in samples without meditative experience (e.g., Baer et al. 2008; Soler et al. 2014a). In the present study, the correlation between observing and the NAS was also the lowest (in both samples) among FFMQ facets. This finding is not surprising bearing in mind some recent studies (e.g., Desrosiers et al. 2014; Williams et al. 2014). For instance, Williams et al. (2014) found that in both an unspecified community adult sample and a clinical sample, the observing facet did not load significantly onto an overarching mindfulness factor, whereas the other four FFMQ facets did.

In the study by Sahdra et al. (2010), associations between NAS and mindfulness measures were studied by testing associations between the NAS and the Mindful Attention and Awareness Scale (MAAS; Brown and Ryan 2003). Such correlations were lower than those reported here between the NAS and the FFMQ. This lower convergence in the original study (Sahdra et al. 2010) may result from the characteristics of the MAAS itself, as it only assesses one component of mindfulness (i.e., lack of attention-awareness), leaving aside the attitudinal/ acceptance facet (Bishop et al. 2004; Brown and Ryan 2003). The FFMQ (Baer et al., 2006) provides information about five mindfulness facets that intend to represent a broader definition of this construct. Interestingly, the lowest correlation was found between the NAS and FFMQ-observing (which is representative of the attention-awareness mindfulness component;
Bergomi et al. 2013). The highest association between NAS scores and FFMQ facets was for nonjudging and nonreactivity subscales. If it is considered that these two scales represent the acceptance component of mindfulness (Baer et al. 2006), it seems that nonattachment could be specially related to this attitudinal element. Coherently, Sahdra et al. (2010) also reported high effect sizes regarding correlations between the NAS and acceptance measured by the Acceptance and Action Questionnaire-II and FFMQ-nonreactivity.

In common with the Sahdra et al. results, we found positive correlations between nonattachment and meditation practice (particularly total weekly hours of meditation practice, and frequency of practice), indicating that such contemplative training, especially when it is done in a regular basis, seems to facilitate the growth of nonattachment. However, given that this study has a cross-sectional design, causality between meditation practice and nonattachment cannot be inferred. Nor can we be sure that those who practice more or maintained practice for longer did not already have higher levels of nonattachment before starting to meditate. Also consistent with Sahdra's results, we found some evidence suggesting that there are other ways of developing nonattachment besides the practice of meditation. Our results indicate that some sociodemographic indices, such as becoming older or having more years of education, seem to foster a nonattached view. One of the possible mechanisms underlying the development of nonattachment seems to be related to a certain kind of wisdom such as an understanding of the impermanent and dependently arising or constructed nature of mental images (Sahdra et al. 2010). It seems that life stressors, losses, and changes, which are inherent to all aspects of life (including schooling), may help individuals to learn how to take a more flexible and open stance regarding objects and experiences.

NAS scores were highly associated with decentering, rather than with FFMQ facets, both in the meditative and the nonmeditative samples. Decentering may be considered as a by-product of mindfulness practice (Tanay et al. 2012) and a psychological quality that can also be enhanced through other psychotherapeutic approaches such as cognitive behavior therapy (Teasdale et al. 2002). It also seems to be fostered by life processes (such as aging and schooling; Soler et al. 2014b). Stronger associations between nonattachment and decentering probably indicate that the former one has more communality with decentering than with mindfulness. In fact, as some authors have pointed out, decentering, or defusion, seems to be a quality embedded in nonattachment (Allen, 2012). It also seems reasonable to consider the existence of some kind of temporality in the development of both capacities, as decentering (i.e., take a present-focused, nonjudgmental stance in regard to thoughts and feelings and to accept them; Fresco et al. 2007b) seems to be a necessary first step or condition in obtaining a detached view of experiences. 
As expected, NAS scores correlated positively and highly with resilience and negatively with depression, anxiety, stress, and difficulties in emotion regulation in both meditative and nonmeditative samples. These findings seem to indicate a prosalutary effect of nonattachment, as they are in accordance with those obtained in the original validation of the scale. Such findings indicate the protective quality of nonattachment against mental afflictions, fostering emotional regulation when facing adversity. Nonattachment would encourage a set of psychological positive qualities (e.g., acceptance, equanimity, compassion, altruism, resilience) and foster a kind of happiness that is not contingent on circumstances (Brown et al. 2007), counterbalancing the inherent suffering of life (Wallace 2005).

The NAS showed high known-group validity since significant differences with moderate-to-strong effect sizes were observed when comparing levels of nonattachment in the meditative, nonmeditative, and BPD groups. Furthermore, the NAS (and the NAS-7) seemed to be sensitive to such differences in a similar way to other well-established and closely related measures such as the FFMQ and the EQ. In contrast to Sahdra et al.'s work (2010), our sample consisted of a more homogeneous sample regarding forms of meditation. The more homogenous meditative sample may also explain the stronger differences regarding NAS scores when compared with subjects naïve to meditation. In the present study, the sample with BPD diagnosis obtained the lowest levels on the NAS, especially when compared with the meditative group. Some studies with BPD samples suggest that patients with this disorder could be especially impaired regarding nonattachment-related variables such as mindfulness (Wupperman, Neumann, Whitman, and Axelrod 2009). Nonattachment seems to be on a continuum, going from individuals with no meditative training dominated by avoiding and grasping tendencies (i.e., attachment) and nonadaptative emotion regulation strategies (as is the case of patients with BPD; Leichsenring et al. 2011) at one extreme, to individuals with training in meditation at the other.

Results derived from hierarchical regression analyses indicate that nonattachment accounts for additional variance when predicting resilience and clinical symptoms. The additional explained variance of nonattachment was modest (but significant) regarding clinical symptoms and more remarkable when predicting resilience. Bearing in mind that the correlations between the NAS and all other variables introduced in the regression models were moderate to high, such an increment in the power of the model is even more noteworthy.

Regression models indicated that being older and having more years of schooling together with some FFMQ facets (i.e., describing, acting with awareness, nonjudging) and decentering would be related to lower levels of psychopathology. It is also worth noting the pathological role of the observing facet when predicting clinical symptoms, probably due to an ambivalent relation between self-focused attention and mental health (Baer et al. 2008). Interestingly, EQ scores were not significant predictors of anxiety, suggesting that the relationship between decentering and anxiety may be fully comprised in the relationship between NAS and DASS-anxiety. A large amount of the variance of CD-RISC-10 scores was predicted by age, observing (negatively associated), describing, the EQ, and especially, the NAS. Resilience involves personal qualities that enable one to thrive in the face of adversity and is also a good measure of successful stress-coping ability (Connor and Davidson 2003). Nonattachment, according to several Buddhism and mindfulness experts, is also expressed in terms of more quickly recovering from upsets (Sahdra et al. 2010), and in this sense, the NAS scale includes items which are in full agreement with the concept of resilience itself, such as "I can accept the flow of events in my life without hanging onto them or pushing them away," or "I can remain open to what life offers me regardless of whether it seems desirable or undesirable at a particular time." Nonattachment is based on insight into the transient nature of mental representations, so a nonattached individual should be less likely to get stuck in self-referential processing when faced with adversity, taking a more self-compassionate and balanced attitude (Desbordes et al. 2015; Sahdra et al. 2010).

\section{Strengths and Limitations of This Study}

The main limitation of the present study is its cross-sectional nature, which impedes the analysis of causality among the NAS and other variables. An Internet-based commercial system was used to recruit the samples, and such sampling methods may be a source of bias (compared with truly random sampling). However, since the study sample was large, the reliability of such method should be adequate (Ritter et al. 2004). All data were obtained by means of self-report instruments which are subject to individual response biases. Studies using larger samples of heterogeneous clinical populations can help to determine whether nonattachment is generally impaired in mental conditions and may also provide insights into the clinical applicability of this construct. Future research should also study the relationship between the Spanish version of the NAS and other close-related relevant concepts in psychotherapy such as selfcompassion, personal values, and eudemonic well-being.

Acknowledgments This study was in part supported by CIBERSAM, CIBEROBN, REDIAPP (Instituto de Salud Carlos III, ISCIII; RD12/ 0005/0006 and RD12/0005/0008), and PROMOSAM (Red de Excelencia PSI2014-56303-REDT). We are also grateful to ISCIII for two grants for research projects on health (PI13/00134 and PI15/00383) that are co-financed with European Union ERDF funds. The third author (JVL) has a Miguel Servet contract awarded by the ISCIII (CP14/00087). 


\section{References}

Aguado, J., Luciano, J. V., Cebolla, A., Serrano-Blanco, A., Soler, J., \& García-Campayo, J. (2015). Bifactor analysis and construct validity of the five facet mindfulness questionnaire (FFMQ) in non-clinical Spanish samples. Frontiers in Psychology, 6, 404. doi:10.3389 /fpsyg.2015.00404.

Allen, J. G. (2012). Mentalizing in the Development and Treatment of Attachment Trauma. Karnac Books

Baer, R. A., Smith, G. T., Hopkins, J., Krietemeyer, J., \& Toney, L. (2006). Using self-report assessment methods to explore facets of mindfulness. Assessment, 13, 27-45.

Baer, R. A., Smith, G. T., Lykins, E., Button, D., Krietemeyer, J., Sauer, S., et al. (2008). Construct validity of the five facet mindfulness questionnaire in meditating and nonmeditating samples. Assessment, 15(3), 329-342. doi:10.1177/1073191107313003.

Bados, A., Solanas, A., \& Andrés, R. (2005). Psychometric properties of the Spanish version of Depression, Anxiety and Stress Scales (DASS). Psicothema, 17, 679-683.

Bandalos, D. L., \& Finney, S. J. (2001). Item parceling issues in structural equation modeling. In G. A. Marcoulides \& R. E. Schumacker (Eds.), Advanced structural equation modeling: new developments and techniques (pp. 269-296). Mahwah: Lawrence Erlbaum.

Barrachina, J., Soler, J., Campins, M. J., Tejero, A., Pascual, J. C., Alvarez, E., et al. (2004). Validation of a Spanish version of the Diagnostic Interview for Bordelines-Revised (DIB-R). Actas Españolas de Psiquiatría, 32, 293-298.

Bergomi, C., Tschacher, W., \& Kupper, Z. (2013). Measuring mindfulness: first steps towards the development of a comprehensive mindfulness scale. Mindfulness, 4(1), 18-32. doi:10.1007/s12671-0120102-9.

Bishop, S. R., Lau, M., Shapiro, S., Carlson, L., Anderson, N. D., Carmody, J., et al. (2004). Mindfulness: a proposed operational definition. Clinical Psychology: Science and Practice, 11, 230-241. doi:10.1093/clipsy.bph077.

Brown, K. W., \& Ryan, R. M. (2003). The benefits of being present: mindfulness and its role in psychological well-being. Journal of Personality and Social Psychology, 84, 822-848.

Brown, K. W., Ryan, R. M., \& Creswell, J. D. (2007). Mindfulness: theoretical foundations and evidence for its salutary effects. Psychological inquiry, 18(4), 211-237. doi:10.1080 /10478400701598298.

Cebolla, A., Garcia-Palacios, R., Soler, J., Guillen, V., Baños, R., \& Botella, C. (2012). Psychometric properties of the Spanish validation of the Five Facets of Mindfulness Questionnaire (FFMQ). European Journal of Psychiatry, 26(2), 118-126. doi:10.4321 /s0213-61632012000200005.

Connor, K. M., \& Davidson, J. R. T. (2003). Development of a new resilience scale: the Connor-Davidson Resilience Scale (CDRISC). Depression and Anxiety, 18, 76-82.

De Bruin, E. I., Topper, M., Muskens, J. G., Bögels, S. M., \& Kamphuis, J. H. (2012). Psychometric properties of the Five Facets Mindfulness Questionnaire (FFMQ) in a meditating and a nonmeditating sample. Assessment, 19(2), 187-197. doi:10.1177 /1073191112446654.

Desbordes, G., Gard, T., Hoge, E. A., Hölzel, B. K., Kerr, C., Lazar, S. W., et al. (2015). Moving beyond mindfulness: defining equanimity as an outcome measure in meditation and contemplative research. Mindfulness, 6(2), 356-372. doi:10.1007/s12671-013-0269-8.

Desrosiers, A., Vine, V., Curtiss, J., \& Klemanski, D. H. (2014). Observing nonreactively: a conditional process model linking mindfulness facets, cognitive emotion regulation strategies, and depression and anxiety symptoms. Journal of Affective Disorders, 165, 31-37. doi:10.1016/j.jad.2014.04.024.
Ferguson, C. J. (2009). An effect size primer: a guide for clinicians and researchers. Professional Psychology: Research and Practice, 40(5), 532. doi:10.1037/a0015808.

Fresco, D. M., Moore, M. T., van Dulmen, M. H., Segal, Z. V., Ma, S. H., Teasdale, J. D., \& Williams, J. M. G. (2007a). Initial psychometric properties of the experiences questionnaire: validation of a selfreport measure of decentering. Behavior Therapy, 38(3), 234-246. doi:10.1016/j.beth.2006.08.003.

Fresco, D. M., Segal, Z. V., Buis, T., \& Kennedy, S. (2007b). Relationship of posttreatment decentering and cognitive reactivity to relapse in major depression. Journal of Consulting and Clinical Psychology, 75(3), 447-455.

Gratz, K. L., \& Roemer, L. (2004). Multidimensional assessment of emotion regulation and dysregulation: development, factor structure, and initial validation of the difficulties in emotion regulation scale. Journal of Psychopathology \& Behavioral Assessment, 26(1), 4154.

Hervás, G., \& Jódar, R. (2008). Adaptación al castellano de la Escala de Dificultades en la Regulación Emocional. Clínica Y Salud, 19(2), 139-156.

Leichsenring, F., Leibing, E., Kruse, J., New, A. S., \& Leweke, F. (2011). Borderline personality disorder. The Lancet, 377(9759), 74-84. doi:10.1016/S0140-6736(10)61422-5.

Lovibond, P. F., \& Lovibond, S. H. (1995). The structure of negative emotional states: comparison of the Depression Anxiety Stress Scales (DASS) with the Beck Depression and Anxiety Inventories. Behaviour Research and Therapy, 33, 335-343.

McIntosh, W. D., \& Martin, L. L. (1992). The cybernetics of happiness: the relation between goal attainment, rumination, and affect. In M. S. Clark (Ed.), Review of Personality and Social Psychology (Vol. 14, pp. 222-146). Newbury Park: Sage Publications.

Notario-Pacheco, B., Solera-Martínez, M., Serrano-Parra, M. D., Bartolomé-Gutiérrez, R., García-Campayo, J., \& MartínezVizcaíno, V. (2011). Reliability and validity of the Spanish version of the 10-item Connor-Davidson Resilience Scale (10-item CDRISC) in young adults. Health and Quality of Life Outcomes, 9, 63. doi:10.1186/1477-7525-9-63.

Ritter, P., Lorig, K., Laurent, D., \& Matthews, K. (2004). Internet versus mailed questionnaires: a randomized comparison. Journal of Medical Internet Research, 6, e29.

Safran, J. D., \& Segal, Z. V. (1990). Interpersonal process in cognitive therapy. New York: The Guilford Press.

Sahdra, B. K., Shaver, P. H., \& Brown, K. W. (2010). A scale to measure nonattachment: a Buddhist complement to Western research on attachment and adaptive functioning. Journal of Personality Assessment, 92(2), 116-127. doi:10.1080/00223890903425960.

Sahdra, B. K., Ciarrochi, J., Parker, P. D., Marshall, S., \& Heaven, P. (2015). Empathy and nonattachment independently predict peer nominations of prosocial behavior of adolescents. Frontiers in Psychology, 6, 263. doi:10.3389/fpsyg.2015.00263.

Schumacher, R. E., \& Lomax, R. G. (2010). A beginner's guide to structural equation modeling (3rd ed.). Mahwah: Lawrence Erlbaum Associates.

Smith, G. T., McCarthy, D. M., \& Anderson, K. G. (2000). On the sins of short-form development. Psychological Assessment, 12, 102-111.

Soler, J., Cebolla, A., Feliu-Soler, A., Demarzo, M. M., Pascual, J. C., Baños, R., et al. (2014b). Relationship between meditative practice and self-reported mindfulness: the MINDSENS composite index. PloS one, 9(1). doi: 10.1371/journal.pone.0086622

Soler, J., Franquesa, A., Feliu-Soler, A., Cebolla, A., García-Campayo, J., Tejedor, R., et al. (2014a). Assessing decentering: validation, psychometric properties and clinical usefulness of the Experiences Questionnaire in a Spanish sample. Behavior Therapy, 45(6), 86371. doi:10.1016/j.beth.2014.05.004.

Steiger, J. H. (1980). Tests for comparing elements of a correlation matrix. Psychological Bulletin, 87, 245-251. 
Tabachnick, B. G., \& Fidell, L. S. (2007). Using multivariate statistics (5th ed.). Pearson Education: Boston.

Tanay, G., Lotan, G., \& Bernstein, A. (2012). Salutary proximal processes and distal mood and anxiety vulnerability outcomes of mindfulness training: a pilot preventive intervention. Behavior therapy, 43(3), 492-505. doi:10.1016/j.beth.2011.06.003.

Teasdale, J. D., Moore, R. G., Hayhurst, H., Pope, M., Williams, S., \& Segal, Z. V. (2002). Metacognitive awareness and prevention of relapse in depression: empirical evidence. Journal of Consulting and Clinical Psychology, 70(2), 275-287. doi:10.1037/0022-006x.70.2.275.

Wallace, B. A. (2005). Genuine happiness: meditation as the path to fulfillment. New York: Wiley.
Williams, M. J., Dalgleish, T., Karl, A., \& Kuyken, W. (2014). Examining the factor structures of the five facet mindfulness questionnaire and the self-compassion scale. Psychological Assessment, 2, 407-418. doi:10.1037/a0035566.

Woods, C. M. (2006). Careless responding to reverse-worded items: implications for confirmatory factor analysis. Journal of Psychopathology and Behavioral Assessment, 28, 186-191. doi:10.1007/s10862-005-9004-7.

Wupperman, P., Neumann, C. S., Whitman, J. B., \& Axelrod, S. R. (2009). The role of mindfulness in borderline personality disorder features. The Journal of nervous and mental disease, 197(10), 766771. doi:10.1097/NMD.0b013e3181b97343. 